Kansas State University Libraries

New Prairie Press

\title{
BAYESIAN ANALYSIS OF DOSE-RESPONSE CALIBRATION CURVES
}

William J. Price

Bahman Shafii

Follow this and additional works at: https://newprairiepress.org/agstatconference

Part of the Agriculture Commons, and the Applied Statistics Commons

\section{(c) $(1) \ominus$}

This work is licensed under a Creative Commons Attribution-Noncommercial-No Derivative Works 4.0 License.

\section{Recommended Citation}

Price, William J. and Shafii, Bahman (2005). "BAYESIAN ANALYSIS OF DOSE-RESPONSE CALIBRATION

CURVES," Conference on Applied Statistics in Agriculture. https://doi.org/10.4148/2475-7772.1135

This is brought to you for free and open access by the Conferences at New Prairie Press. It has been accepted for inclusion in Conference on Applied Statistics in Agriculture by an authorized administrator of New Prairie Press. For more information, please contact cads@k-state.edu. 


\title{
BAYESIAN ANALYSIS OF DOSE-RESPONSE CALIBRATION CURVES
}

\author{
William J. Price, Bahman Shafii \\ Statistical Programs \\ College of Agricultural and Life Sciences \\ University of Idaho, Moscow, ID
}

The statistical analysis of dose-response experiments typically models observed responses as a function of an applied dosage series. The estimated "dose-response curve" is used in predicting future responses, however, it is also commonly rewritten in an inverted form where dose is expressed as a function of the response. This modified "calibration curve" is useful in cases where observed responses are available, but their associated dosages are unknown. Traditional statistical techniques for the estimation of unknown doses from the dose-response curve are problematic, involving approximate solutions and methods. Alternatively, this type of inverse calibration problem naturally falls into the framework of Bayesian analysis. That is, one wishes to estimate the probability of an unknown dose value at an observed value of the response given the underlying relationship between the dose and response. This paper examines some potential Bayesian solutions to the calibration problem under various assumptive conditions. The required methodology in each case will be outlined for a dichotomous response variable and a logistic dose-response function. Empirical results will be demonstrated using data from an organic pesticide dose-response trial.

Keywords: Binomial Response, Logistic Function, Bayesian Estimation

\section{INTRODUCTION}

Dose-response problems are common in agricultural and biological research and can readily be found in plant, soil, entomological, and animal sciences as well as microbiology, clinical, and pharmaceutical fields. Defining dose-response relationships is useful for assessing the effects of controlled experimental factors (dose) on measured responses. These problems can be generalized to include the effects of time on a response, e.g. seed germination, insect emergence; or chemical degradation as well as the more obvious settings which describe the effects of chemical exposure rates on a response. In most situations, analyses include modeling the response as a function of the dosage factor. The function chosen is typically nonlinear and usually drawn from the family of increasing or decreasing sigmoidal curves (probit, logistic, and Gompherz, for example), although asymptotic curves such as exponential or hyperbolic forms are also employed. Measured responses may be either continuous or discrete and parameter estimation is accomplished by using either linearized models or iterative nonlinear methods.

A problem related to the dose-response estimation is the bioassay. Bioassays typically involve the development of a standard or calibration curve which is subsequently used to adjust previously measured values or determine unknown quantities. For the later case, the researcher 
measures a response for which the associated dose is unknown. Of practical concern is determining what dose generated the observed response. This question can be naturally posed in terms of Bayes Theorem: "What is the probability of a specified dose given the observed response and the dose-response curve?" This paper will outline and compare two Bayesian methods for estimating an unknown dose given a response assuming: 1) That the dose-response curve is known (deterministic) and 2) that the dose response curve is estimated. Although the general methods outlined below are applicable to any dose-response function, the material given will concentrate on a binomial response in conjunction with a logistic dose-response model.

\section{METHODS}

\section{Bayesian Dose-Response Estimation}

The logistic model, introduced by Berkson (1944), provides an easily interpreted framework for dose-response modeling and is commonly used in such analyses. In this case, the response of the $\mathrm{j}^{\text {th }}$ replication for the $\mathrm{i}^{\text {th }}$ level of dose, $\mathrm{y}_{\mathrm{ij}}$, is assumed to be distributed as a binomial variate. The entire data vector is denoted by $\mathbf{y}$ where $\mathbf{y}=\left(\mathrm{y}_{11}, \mathrm{y}_{12}, \ldots, \mathrm{y}_{\mathrm{IJ}}\right)$ and the proportion of success for the $\mathrm{i}^{\text {th }}$ dose, $\mathrm{x}_{\mathrm{i}}$, is given by:

$$
\pi_{i}=\frac{1}{1+\exp \left(-\beta\left(x_{i}-\gamma\right)\right)}
$$

where $\beta$ is a rate related parameter and $\gamma$ is the dose for which the proportion of success is 0.5 .

The posterior distribution for $\theta=[\beta, \gamma]$ is given by Bayes formula:

$$
p(\boldsymbol{\theta} \mid \boldsymbol{y})=\frac{p(\boldsymbol{y} \mid \boldsymbol{\theta}) \cdot p(\boldsymbol{\theta})}{\int_{\theta} p(\boldsymbol{y} \mid \boldsymbol{\theta}) \cdot p(\boldsymbol{\theta}) d_{\theta}}
$$

where $\mathrm{p}(\mathbf{y} \mid \theta)$ is a likelihood for the data $\mathbf{y}$ evaluated over the parameters $\theta$, and $\mathbf{p}(\theta)$ is a prior distribution for the parameters $\theta$. The likelihood, based on the binomial, is given as:

$$
L(\boldsymbol{\theta} \mid \boldsymbol{y}) \equiv p(\boldsymbol{y} \mid \boldsymbol{\theta}) \propto \underset{i=1}{\amalg} \prod_{j=1}^{I}\left(\pi_{i}\right)^{y_{i j}}\left(1-\pi_{i}\right)^{N_{i j}-y_{i j}}
$$

The prior probabilities for $\beta$ and $\gamma$ must be user-specified, however, the upper bound on $\beta$ is open ended, i.e. it can be infinite. The bounds on $\gamma$ may also be considered unbounded. In order to accommodate easier prior specification, the logistic model given in (1) can be reparameterized (Price, et al., 2003). Specifically, when the dose level is set to zero or the dose level is set to the maximum dose tested, $\mathrm{x}_{\max }$, the logistic model reduces to:

$$
\theta_{0}=\frac{1}{1+\exp (\beta \cdot \gamma))} \text { and } \theta_{\max }=\frac{1}{1+\exp \left(-\beta \cdot\left(x_{\max }-\gamma\right)\right)}
$$

where $\theta_{0}$ and $\theta_{\max }$ are the proportions of success at dose $=0$ and dose $=x_{\max }$, respectively. Solving (4) for $\beta$ and $\gamma$ yields:

$$
\begin{aligned}
& \gamma=\frac{x_{\max } \cdot \ln \left(\left(1-\theta_{0}\right) / \theta_{0}\right)}{\ln \left(\left(1-\theta_{0}\right) / \theta_{0}\right)-\ln \left(\left(1-\theta_{\max }\right) / \theta_{\max }\right)} \\
& \beta=\ln \left(\left(\left(1-\theta_{0}\right) / \theta_{0}\right) / \gamma\right)
\end{aligned}
$$


which can be used to specify the logistic model (1) in terms of $\theta_{0}$ and $\theta_{\max }$. The proportions $\theta_{0}$ and $\theta_{\max }$ take on values between 0 and 1. For prior specification, we can apply the principle of maximum entropy (Jaynes, 2003) to define uniform prior probability distributions for $\theta_{0}$ and $\theta_{\max }$ as $\mathrm{p}\left(\theta_{0}\right)=\mathrm{p}\left(\theta_{\max }\right)=$ beta[1,1]. Furthermore, assuming independence of $\theta_{0}$ and $\theta_{\max }$, the joint probability distribution of $\theta$ is then $p(\theta)=p\left(\theta_{0}\right) \bullet p\left(\theta_{\text {max }}\right)$. The reparameterized posterior distribution (2) can then be written as:

$$
p\left(\theta_{0}, \theta_{\max } \mid y\right)=\frac{p\left(y \mid \theta_{0}, \theta_{\max }\right) \cdot p\left(\theta_{0}\right) \cdot p\left(\theta_{\max }\right)}{\int_{\theta_{0}} \int_{\theta_{\max }} p\left(y \mid \theta_{0}, \theta_{\max }\right) \cdot p\left(\theta_{0}\right) \cdot p\left(\theta_{\max }\right) d_{\theta_{0}} d_{\theta_{\max }}}
$$

The marginal distributions for $\theta_{0}$ and $\theta_{\max }$ can be found through integration of the posterior distribution in (6) using numerical or simulation (Monte Carlo) techniques. Given these marginal distributions, the associated distributions and point estimates for $\beta$ and $\gamma$ can also be found using the relationships in (5).

\section{Estimation of an Unknown Dose}

(i) Dose-response curve with known parameters

Given a new observation of $\mathrm{M}$ successes from $\mathrm{N}$ trials at an unknown dose, and assuming the logistic dose-response curve shown in (1) with the parameters $\theta_{0}$ and $\theta_{\max }$ known, the probability that the unknown dose $=\mathrm{x}$ given the observed proportion of success, $\mathrm{M} / \mathrm{N}$, is :

$$
p\left(x \mid M, N, \theta_{0}, \theta_{\max }\right) \propto p\left(M \mid x, N, \theta_{0}, \theta_{\max }\right) \cdot p(x)=\pi^{M}(1-\pi)^{N-M} p(x)
$$

where $\mathrm{p}(\mathrm{x})$ is a prior probability for $\mathrm{x}$. Assuming a uniform distribution for $\mathrm{x}$ within the calibration range of doses, a closed form solution for the point estimate of $\mathrm{x}$ is:

$$
\hat{x}=\left(\ln \left(\frac{M}{N-M}\right) / \beta\right)+\gamma
$$

and a $(1-\alpha) \%$ credible interval for $\mathrm{x}$ can be derived from the posterior distribution (7) using:

$$
p(L \leq x \leq U)=1-\alpha
$$

where $\mathrm{L}$ and $\mathrm{U}$ are the upper and lower percentiles, respectively, of the posterior distribution given in (7).

\section{(ii) Dose-response curve known with estimated parameters}

This scenario is similar to (i) above, however it relaxes the assumptions on the doseresponse curve by incorporating its error of estimation into the problem. Hence, we assume that 1) $M$ successes in $N$ trials at an unknown dose have been observed, 2) the logistic model (1) is used, and 3 ) the parameters $\theta_{0}$ and $\theta_{\max }$ are estimated from a set of calibration data, $\left[\mathrm{y}_{\mathrm{ij}}, \mathrm{x}_{\mathrm{i}}\right]$.

If the observed $\mathrm{M}$ successes are independent of the calibration responses, $\mathbf{y}$, and the unknown dose, $\mathrm{x}$, is independent of the parameters, $\theta_{0}$ and $\theta_{\max }$, then the posterior density 
function for the unknown dose can be derived from $\mathrm{p}(\mathrm{M} \mid \mathrm{x})$ and $\mathrm{p}\left(\theta_{0}, \theta_{\max } \mid \mathbf{y}\right)$ as:

$$
p(x \mid M, N, y) \propto \int_{\theta_{0}} \int_{\theta_{\max }} p(M \mid x) \cdot p(x) \cdot p\left(\theta_{0}, \theta_{\max } \mid y\right) d_{\theta_{0}} d_{\theta_{\max }}
$$

where $\mathrm{p}(\mathrm{M} \mid \mathrm{x})$ is given by $\pi_{\mathrm{i}}^{\mathrm{M}}\left(1-\pi_{\mathrm{i}}\right)^{\mathrm{N}-\mathrm{M}}, \mathrm{p}(\mathrm{x})$ is the prior distribution for the unknown dose, $\mathrm{x}$, and $\mathrm{p}\left(\theta_{0}, \theta_{\max } \mid \mathbf{y}\right)$ is the posterior distribution given in (6). In essence, this filters the posterior distribution above in (7) through the posterior distribution from the dose-response curve estimation.

Using a predefined prior distribution for $\mathrm{x}$, a solution for the unknown dose as well as the associated $(1-\alpha) \%$ credible intervals can be found from (10) using numerical or simulation techniques.

All programs and graphics were carried out using SAS (2001). Sample programs and data are available at: http://www.uidaho.edu/ag/statprog .

\section{EMPIRICAL RESULTS}

The data used to demonstrate the methods outlined above represent the effects of an organic pesticide on the egg hatch of black vine weevil (BVW). This organism provides a good biological model for assessing the efficacy of the pesticide. In the dose-response experiment, 20 eggs were placed in a petri dish with a fixed dose of the pesticide. Nine doses (concentrations) were used ranging from 0.0 to $0.03 \mathrm{~g}$. Each dose was replicated 10 times and the experiment was repeated three times. The number of eggs failing to hatch (unhatched) out of the 20 possible was recorded as a success.

The logistic model given in (1) provided a good fit to the data (Figure 1). Both parameters, $\theta_{0}$ and $\theta_{\max }$, were significant and the $95 \%$ credible regions reasonable. The rate of unhatched eggs at dose $=0.0 \mathrm{~g}, \theta_{0}$, was estimated at 0.0175 while the final unhatched rate, $\theta_{\max }$, was essentially $100 \%$ at 0.9999 . Translated into the natural parameters of the logistic function, $[\gamma, \beta]$ gave an inflection point of $\gamma=0.0086 \mathrm{~g}$ and a rate parameter estimate of $\beta=466.8$ (Table $1)$.

\section{(i) Dose-response curve with known parameters}

Assuming the logistic model and the point estimates given above and three "new" observed unhatched rates $(5 / 20,10 / 20$, and 19/20), the most probable doses were estimated. The prior distribution for the unknown dose in each case was assumed uniform within the range of doses tested, i.e. U[0.0, 0.03]. The posterior distributions, most probable values, and $95 \%$ credible regions obtained through numerical methods are given in Figure 2. As the mortality rates approach 0.0 or 1.0, the posterior distributions for the unknown doses become skewed, while that of the $50 \%$ rate is symmetrical. Each of these scenarios assumed 20 trials. The effects of increasing the number of trials on the posterior distribution is demonstrated in Figure 3, while maintaining the rate of unhatched eggs at $50 \%$. As expected, the dispersion of the distribution decreases as the number of trials increases from 20 to 100 . Hence, increasing the number of trials used to determine an unknown dose will increase the precision of the final estimate. Such a 
sensitivity analysis could prove useful for determining desired precision and sample sizes for estimating unknown doses.

\section{(ii) Dose-response curve with estimated parameters}

While the method demonstrated above provides estimates of the unknown dose, it fails to account for the variability associated with the calibration curve estimation. As a means of incorporating that variability, the estimated posterior distribution of the calibration parameters, (6), was used as shown in (10) to obtain the unknown dose posterior for 10/20 successes. The uniform prior was again assumed for dose. Figure 4 shows this posterior distribution in comparison to the previous estimation. The distribution and point estimate appear similar to the results above with slightly wider credible limits: 0.0071 to 0.0104 . Although accounting for the calibration curve error did increase the error of the final dose estimation, the effect was not as prominent as might be expected. Examination of the calibration data provides some explanation. The calibration data, as shown in Figure 1, represents 1580 trials which leads to a very high level of precision for the calibration curve. In order to reduce this precision, data points were randomly selected from each dose of the original data to give a total of 310 trials. The doseresponse curve was then re-estimated and the posterior dose distributions above were recomputed using this subset data. These results are shown in Figure 5. The credible regions are wider when the calibration error is incorporated and the point estimates remain unchanged.

While interval estimates provide some insight into the difference between the two distributions, it would be more useful to quantify this difference. Shannon's Entropy (Shannon, 1948) provides a measure of information for probability distributions:

$$
H=-\sum_{i} p\left(x_{i}\right) \cdot \ln \left(p\left(x_{i}\right)\right)
$$

where $\mathrm{p}\left(\mathrm{x}_{\mathrm{i}}\right)$ represents the posterior probability of the $\mathrm{i}^{\text {th }}$ level of dose. By taking the ratio of entropy values from two distributions, a measure of relative information can be defined as: $E_{R}=H_{1} / H_{2}$. If $\mathrm{H}_{2}$ represents the entropy of the "calibration curve known" distribution, i.e. method (i), and $\mathrm{H}_{1}$ the "parameters estimated" distribution, method (ii), the value of $\mathrm{E}_{\mathrm{R}}$ will measure the relative efficiency or distance from method ii) compared to the "perfect" information of method (i). For the example of 10/20 successes, the efficiency is $E_{R}=0.939$. As the number of trials used to determine the unknown dose is increased, however, the efficiency drops (Table 2). This occurs because the error from the calibration curve estimation eventually becomes larger relative to the precision in the unknown dose posterior distribution. Hence, increasing the sampling effort for the unknown dose can not overcome the underlying variability in the calibration curve. Therefore, when estimation of unknown doses is of concern, it is prudent to focus sampling effort and resources on the development of the calibration curve.

\section{Posterior Distribution Behavior}

The examples above have concentrated on the case where $50 \%$ success is observed. Figure 6 demonstrates the differences seen between the methods (i) and (ii) at a higher rate of success, say 19/20. Most notable is the increase in the most probable value (point estimate) from 
method (i) to method (ii). This is due to an increase in distribution skewness, most likely caused by the curvature of the likelihood in (6) at higher values of $\pi_{\mathrm{i}}$. A similar shift in skewness, but in the opposite direction, can be shown for low success rates.

At more extreme success rates, e.g. $0 \%$ and $100 \%$, the distributional behavior is more unusual. Under these conditions, the distributions become open ended with no lower bounds in the case of 0 successes and no upper bound with $100 \%$ success. Figure 7 demonstrates the later case with 20/20 successes. The unknown dose is estimated at 0.03 , the maximum possible, with a lower $95 \%$ credible limit of 0.0138 . This is interpreted as follows: When $100 \%$ of the BVW eggs fail to hatch, the best guess for the associated dose is $0.03 \mathrm{~g}$, however, the dose could be as low as $0.0138 \mathrm{~g}$. In cases with zero successes, the estimated dose would be $0.0 \mathrm{~g}$ with an upper bound representing the largest possible dose that could be present.

\section{CONCLUDING REMARKS}

Dose-response estimation is widely used in scientific research and often involves calibration curves used to determine unknown doses through bioassay studies. The bioassay problem is naturally posed as a Bayesian estimation problem. Estimation of an unknown dose can be carried out using the calibration curve in a deterministic fashion or by incorporating the error inherent from the estimation. Using the later approach will decrease the precision of the final dose estimation. However, the degree to which this may occur will depend on the amount of information used for estimating the calibration curve. Increasing sampling effort for the unknown dose can not overcome the error associated with the calibration curve. Therefore, it is important to concentrate sampling resources on development of the calibration curve, rather than the measurement of the unknown dosages.

\section{REFERENCES}

Berkson, J. 1944. Application of the Logistic function to bio-assay. J. Amer. Stat. Assoc. 39, pp 357-65.

Jaynes, E. T. 2003. Probability Theory. Cambridge University Press, Cambridge, UK. pp. 727.

Price, W. J., B. Shafii, K. B. Newman, S. Early, J. P. McCaffrey, M. J. Morra. 2003. Comparing Estimation Procedures for Dose-response Functions. In Proceedings of the Fifteenth Annual Kansas State University Conference on Applied Statistics in Agriculture [CD-ROM], Kansas State University, Manhattan, KS, pp. 82-99.

SAS Inst. Inc. 2001. SAS Online Doc, Version 8, Cary, NC.

Shannon, C., 1948. The Mathematical Theory of Communication. Bell System Technical Journal, 27: 379, 623. 
Table 1. Parameter estimates and $95 \%$ credible regions for the logistic model fitted to the Black Vine Weevil data.

\begin{tabular}{|c|c|c|c|}
\hline \multirow[b]{2}{*}{ Parameter } & \multirow[b]{2}{*}{$\underline{\text { Estimate }}$} & \multicolumn{2}{|c|}{ 95\% Credible Region } \\
\hline & & Lower & Upper \\
\hline$\theta_{0}$ & 0.01750 & 0.01280 & 0.02320 \\
\hline$\theta_{\max }$ & 0.99995 & 0.99990 & 0.99998 \\
\hline$\gamma$ & 0.00864 & 0.00832 & 0.00891 \\
\hline$\beta$ & 466.8000 & 423.547 & 502.796 \\
\hline
\end{tabular}

Table 2. Relative efficiencies $\left(E_{R}\right)$ of various sample sizes. Proportion of success held is constant at $50 \%$.

\begin{tabular}{|l|l|l|}
\hline $\begin{array}{c}\text { Observed } \\
\text { Successes }\end{array}$ & \multicolumn{1}{|c|}{$\begin{array}{c}\text { Total \# } \\
\text { Trials }\end{array}$} & $\mathbf{E}_{\mathbf{R}}$ \\
\hline 10 & 20 & 0.939 \\
\hline 25 & 50 & 0.913 \\
\hline 50 & 100 & 0.876 \\
\hline 400 & 800 & 0.670 \\
\hline
\end{tabular}




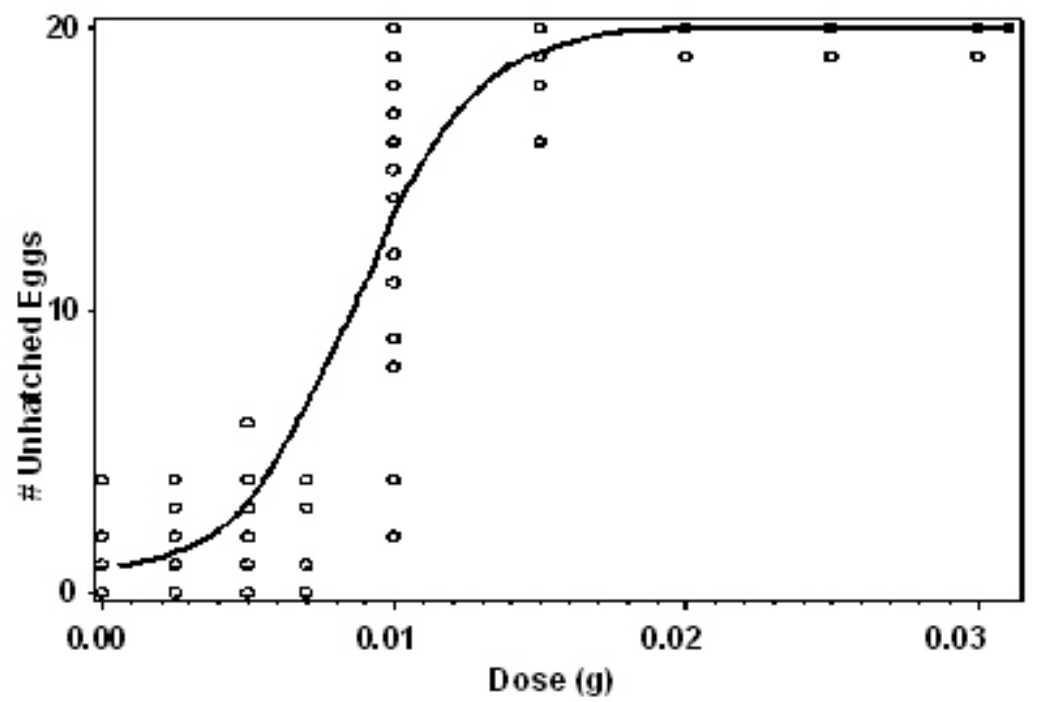

Figure 1. Estimated logistic curve fitted to the Black Vine Weevil data.

a

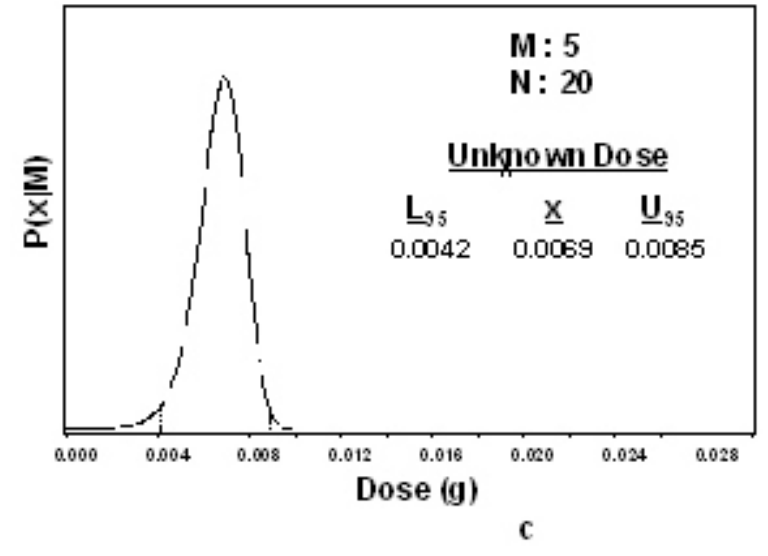

b

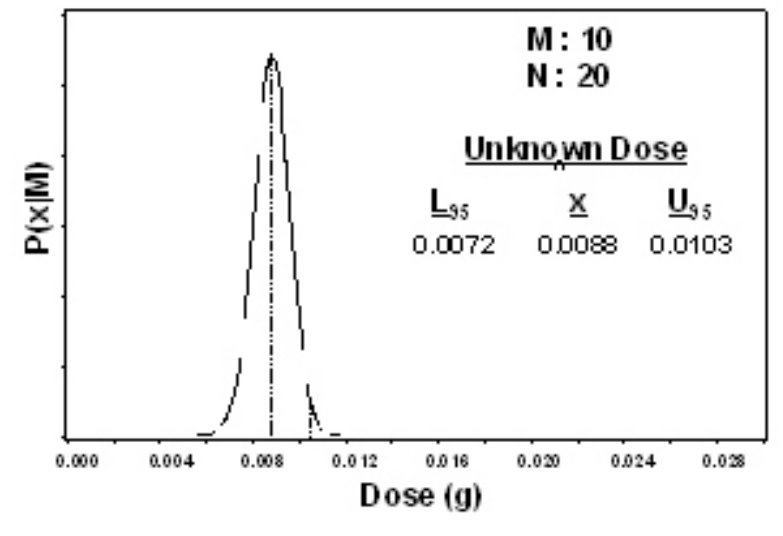

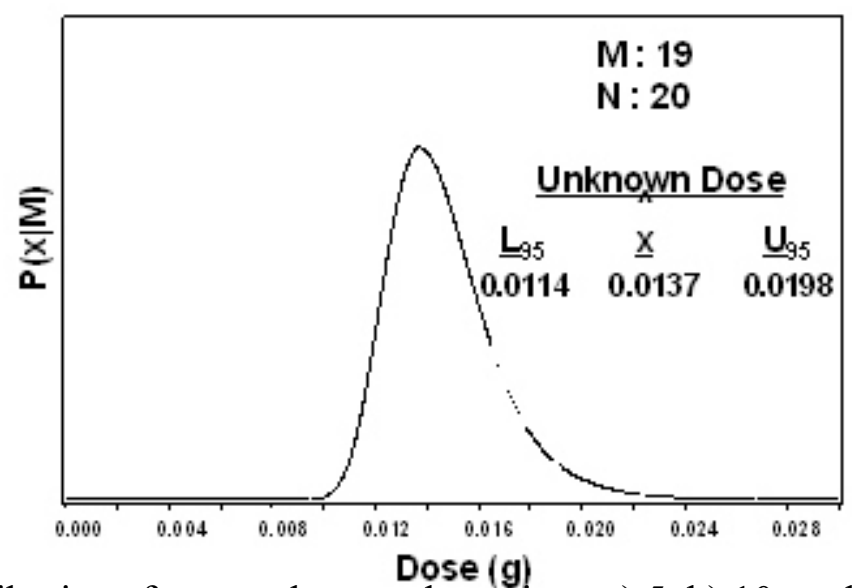

$M: 19$

$\mathrm{N}: 20$

Figure 2. Posterior distributions for an unknown dose given a) 5, b) 10, and c) 19 successes out of 20 trials. Lower (L) and upper (U) 95\% credible intervals along with most probable values are also indicated. 


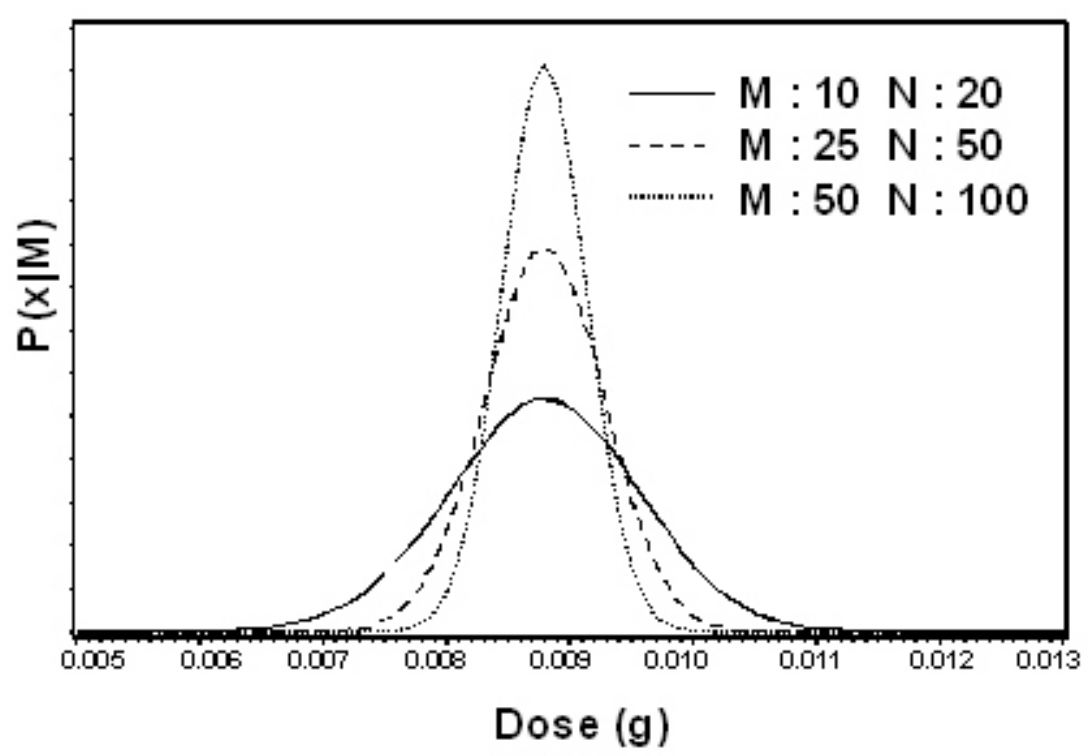

Figure 3. Posterior distribution for an unknown dose given a $50 \%$ success rate under various sampling intensities.

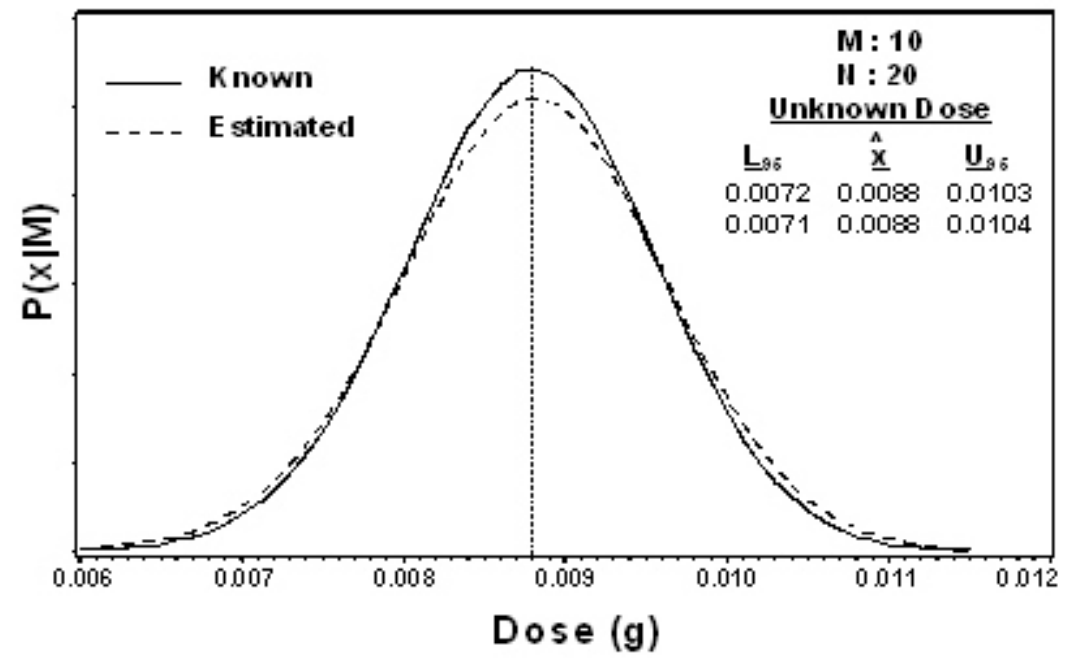

Figure 4. Posterior distributions of an unknown dose given a 50\% success rate. Solid curve represents estimation with the dose-response curve known, while the dashed curve represents estimation with the dose-response curve estimated. Estimation is carried out using the complete Black Vine Weevil data set (1580 observations). 


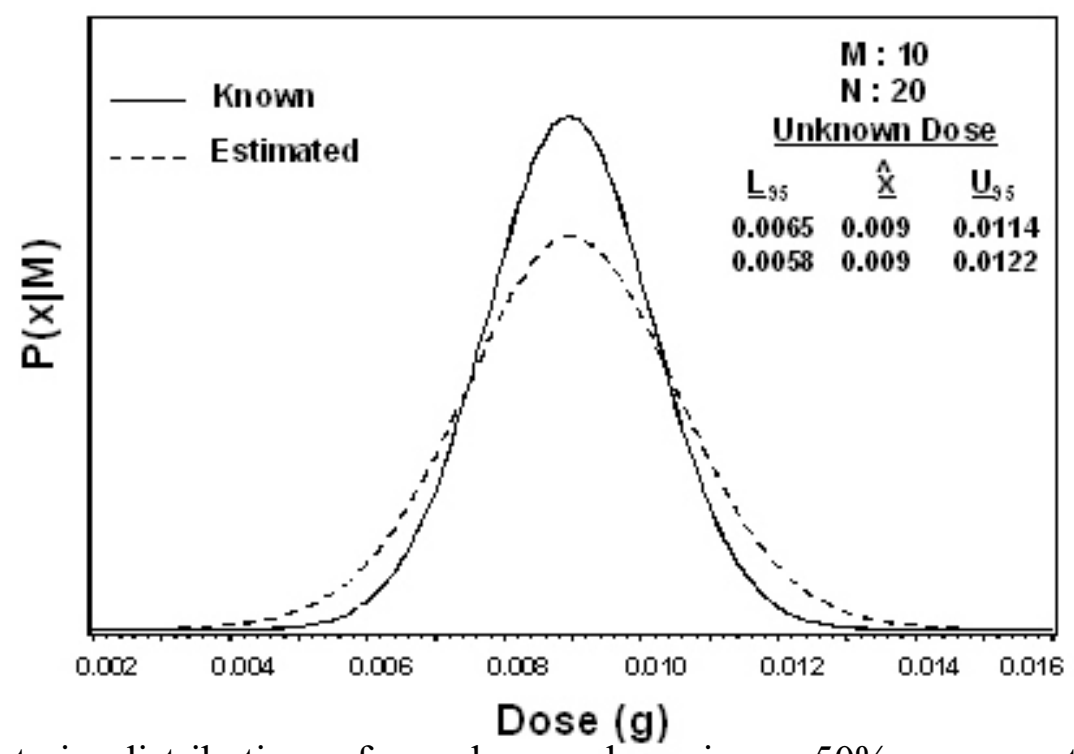

Figure 5. Posterior distributions of an unknown dose given a 50\% success rate. Solid curve represents estimation with the dose-response curve known, while the dashed curve represents estimation with the dose-response curve estimated. Estimation is carried out with a partial Black Vine Weevil data set (310 observations).

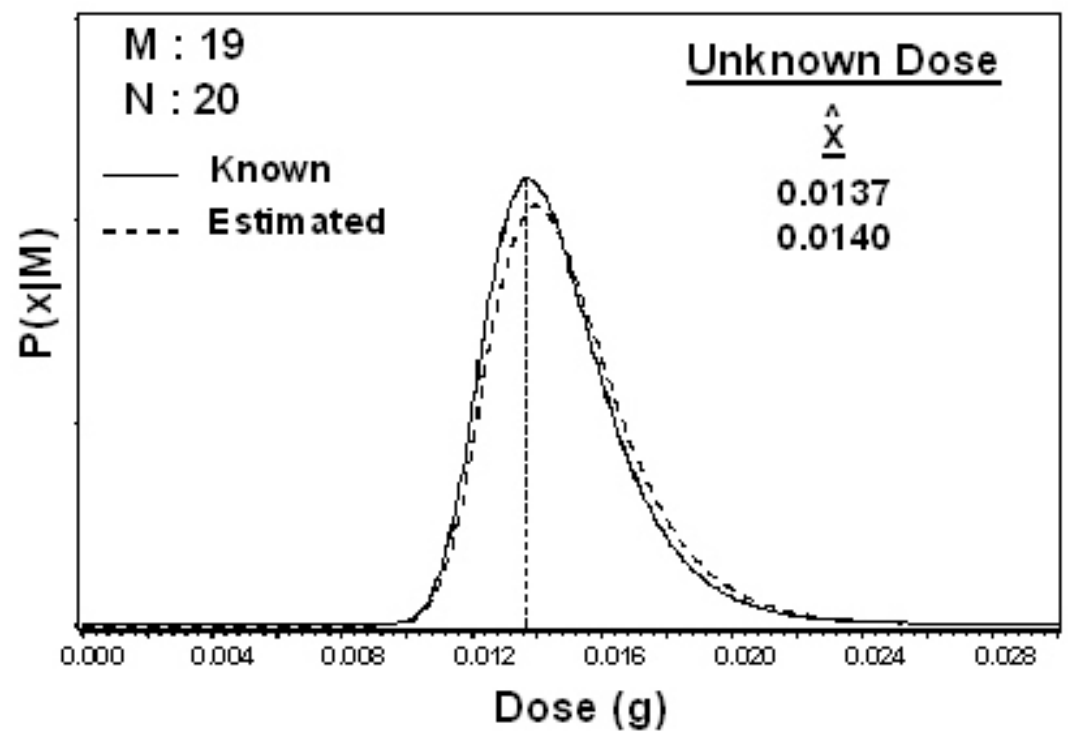

Figure 6. Posterior distributions of an unknown dose given 19/20 successes. Solid curve represents the case with the dose-response curve known, while the dashed curve represents the case where the dose-response curve is estimated. 


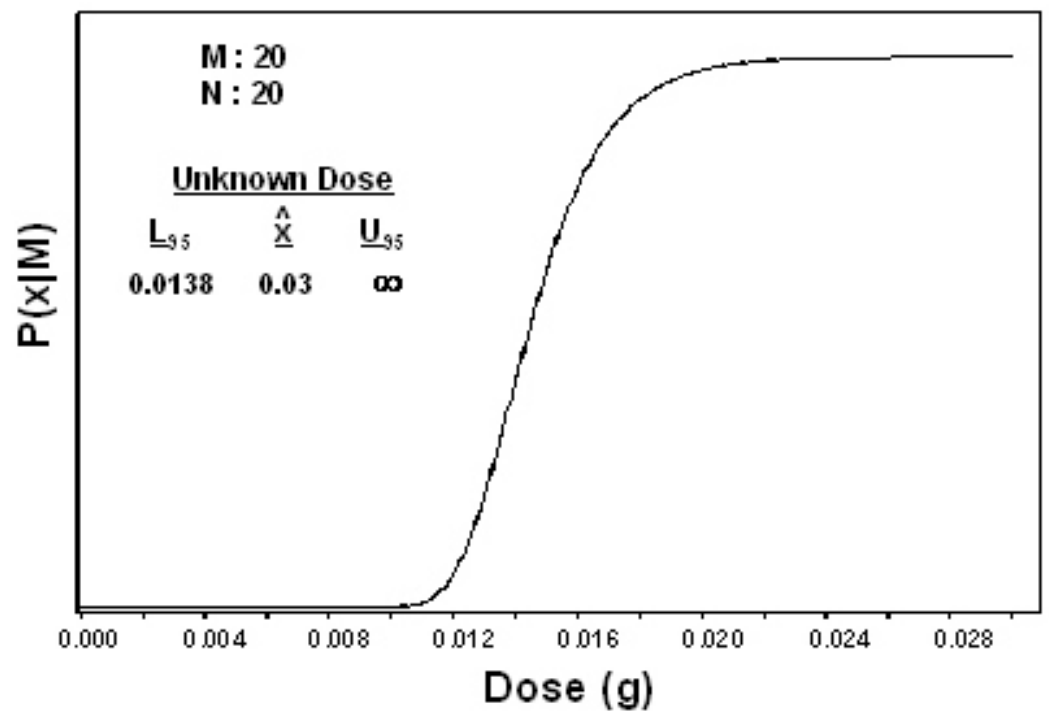

Figure 7. Posterior distributions of an unknown dose given a $100 \%$ success rate. Lower (L) and upper (U) 95\% credible intervals as well as the most probable value are also provided. 